\title{
DETECTION OF THE POSITION OF TWO BEAMS WITH A COMMON BPM
}

\author{
Kotaro SATOH, KEK, Tsukuba, Ibaraki, Japan
}

\begin{abstract}
This paper proposes a method of detecting the position of two beams with a common BPM, the method which is useful even if the bunch time-spacing between the two beams is too small to separate each beam signal with fast switches. The method is based on the idea that unknown beam parameters can be estimated if the number of BPM electrodes is greater than that of the parameters, and if the signals are independent of each other. Since the number of unknown parameters of the two beams is 6 , consisting of 4 positions and 2 intensities, an 8-electrode BPM, whose signals are detected in the frequency domain, is expected to play the role. The method is to be applied to measuring beam positions near the interaction point of the two-ring collider $\mathrm{KEKB}$, where the bunch spacing is only $2 \mathrm{~ns}$ in each ring. The independence of the signals is insured by a finite orbit separation at the common BPM.
\end{abstract}

\section{INTRODUCTION}

In a two-ring collider like the KEKB orbit stabilities at the interaction point are essential for keeping stable beam collisions. Near the interaction point the orbit separation is so small that the two beams travel through common pipes and common BPMs, if inserted. If position measurements are required with the common BPM, it has been believed that the bunch separation, in the time domain, between the two beams must be sufficiently large for separating beam signals with fast switches. In the KEKB, however, the bunch spacing is too small to apply the above method. It should be pointed out that, although the present case has not a sufficient separation in the time domain, here exists a finite orbit separation between the two beams. Providing a finite orbit separation in the transverse space, the paper discusses a possibility of detecting the beam position of each ring with a common BPM having many electrodes. The present method is an extension of that in the previous papers[1][2].

\section{OUTPUT SIGNAL MODEL}

One of the common BPMs, analyzed here, is $55 \mathrm{~mm}$ in diameter, and has 8 electrodes, as shown in Fig.1. The ideal orbits of the two rings, $(-7.5,0)$ for the positron LER and $(6.26,0)$ for the electron HER, are shown in the same figure. The horizontal orbit separation at the BPM is $13.8 \mathrm{~mm}$. The output signal is detected with a narrow-band detector at $1017.16 \mathrm{MHz}$, two times the accelerating frequency.

The output signal can be represented well with a phaser. The phaser output of the i-th pickup button $\tilde{V}_{i}$ is given by

$$
\tilde{V}_{i}=g_{i} e^{i \phi_{i}}\left(p F_{i}(x, y)+q F_{i}(u, v) e^{i \theta}\right), i=1, \ldots, 8
$$

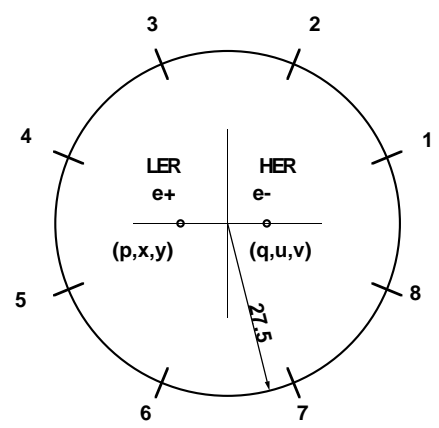

Figure 1: Geometry of the model BPM and the design orbits.

where $g_{i}, \phi_{i}$ and $F_{i}(x, y)$ are the gain, the phase shift and the response function of the i-th electrode, $(p, x, y),(q, u, v)$ are the charge and position for positron and electron beams, and $\theta$ their phase difference.

The detector measures the peak value $V_{i}$ of the phaser,

$V_{i}=g_{i} \sqrt{\left(p F_{i}(e+)\right)^{2}+\left(q F_{i}(e-)\right)^{2}+2 p q F_{i}(e+) F_{i}(e-) \cos \theta}$.

The gains can be calibrated with beams, as a later section shows. The response function is calculated from the BPM geometry. The phase difference $\theta$ must be constant and can be calculated from the distance between the BPM and the interactionpoint, or can be estimated with beams as shown later. For each measurement are 6 unknown parameters $(p, x, y, q, u, v)$. Since the number of unknown parameters is less than that of the BPM electrodes, the parameters are determined by nonlinear fitting. It should be pointed out here that, if the phase were included into fit parameters, the phase resolution would be very poor and would degrade the other resolutions accordingly. By analyzing the covariant matrix associated with the fitting procedure, we can esimate the errors of fit parameters for given measurement errors. The expected error of the $\mathrm{i}$-th parameter $\sigma_{i}$ is given by the $\mathrm{i}$-th diagonal element of the covariant matrix $C(i, j)$. Text books show

$$
\sigma_{i}^{2}=C(i, i) \times \sigma_{0}^{2},
$$

where $\sigma_{0}$ is the absolute measurement error of signals.

The position response function used here is derived from a simple BPM model having very small electrodes. More realistic response functions can be used, if needed. The response functions are expanded with harmonic functions of positions up to the 4-th order terms, and are given by

$$
F_{i}(X, Y)=1+\sum_{k=1}^{4} R^{k}\left(a_{i}(k) \cos k \varphi+b_{i}(k) \sin k \varphi\right),
$$


Table 1: Fit parameters

\begin{tabular}{r|rrrrrr}
$\mathrm{j}$ & $\mathrm{p}$ & $\mathrm{x}$ & $\mathrm{y}$ & $\mathrm{q}$ & $\mathrm{u}$ & $\mathrm{v}$ \\
\hline $\mathrm{1}$ & 2.29 & -7.50 & .00 & -1.00 & 6.26 & .00 \\
2 & 2.29 & -5.50 & .00 & -1.00 & 6.26 & .00 \\
3 & 2.29 & -7.50 & 2.00 & -1.00 & 6.26 & .00 \\
4 & 2.29 & -9.50 & .00 & -1.00 & 6.26 & .00 \\
5 & 2.29 & -7.50 & -2.00 & -1.00 & 6.26 & .00 \\
6 & 2.29 & -7.50 & .00 & -1.00 & 8.26 & .00 \\
7 & 2.29 & -5.50 & .00 & -1.00 & 8.26 & .00 \\
8 & 2.29 & -7.50 & 2.00 & -1.00 & 8.26 & .00 \\
9 & 2.29 & -9.50 & .00 & -1.00 & 8.26 & .00 \\
10 & 2.29 & -7.50 & -2.00 & -1.00 & 8.26 & .00 \\
11 & 2.29 & -7.50 & .00 & -1.00 & 6.26 & 2.00 \\
12 & 2.29 & -5.50 & .00 & -1.00 & 6.26 & 2.00 \\
13 & 2.29 & -7.50 & 2.00 & -1.00 & 6.26 & 2.00 \\
14 & 2.29 & -9.50 & .00 & -1.00 & 6.26 & 2.00 \\
15 & 2.29 & -7.50 & -2.00 & -1.00 & 6.26 & 2.00 \\
16 & 2.29 & -7.50 & .00 & -1.00 & 4.26 & .00 \\
17 & 2.29 & -5.50 & .00 & -1.00 & 4.26 & .00 \\
18 & 2.29 & -7.50 & 2.00 & -1.00 & 4.26 & .00 \\
19 & 2.29 & -9.50 & .00 & -1.00 & 4.26 & .00 \\
20 & 2.29 & -7.50 & -2.00 & -1.00 & 4.26 & .00 \\
21 & 2.29 & -7.50 & .00 & -1.00 & 6.26 & -2.00 \\
22 & 2.29 & -5.50 & .00 & -1.00 & 6.26 & -2.00 \\
23 & 2.29 & -7.50 & 2.00 & -1.00 & 6.26 & -2.00 \\
24 & 2.29 & -9.50 & .00 & -1.00 & 6.26 & -2.00 \\
25 & 2.29 & -7.50 & -2.00 & -1.00 & 6.26 & -2.00 \\
\hline
\end{tabular}

where $X=R \cos \varphi, Y=R \sin \varphi$, and

$$
a_{i}(k)=2 \cos \left(k(2 i-1) \frac{\pi}{8}\right), b_{i}(k)=2 \sin \left(k(2 i-1) \frac{\pi}{8}\right) .
$$

The function is normalized by $F_{i}(0,0)=1$, and the position is measured from the BPM center, not from the ideal orbit.

\section{SIMULATION}

Simulation was done for a case where the position of each beam is on the ideal orbit, or shifted by $\pm 2 \mathrm{~mm}$ at each measurement. Measurement is done 25 times with different position combination. The phase difference $\theta$ is $\pi / 4$. The nominal charge of each ring is $\mathrm{p}=2.29$ for LER and $\mathrm{q}=-1.0$ for HER. The optimum charge ratio is determined by the energy ratio of two rings, $3.5 \mathrm{GeV}$ and $8.0 \mathrm{GeV}$.

The objective of the simulation is not only the demonstration of measuring the two beam positions, but also the derivation of the covariant matrix to estimate the position resolution. Fit parameters for 25 measurements are listed in Table 1, and the diagonal elements of the covariant matrix are shown in Table 2 . Assuming a relative signal measurement error of $3 \times 10^{-4}$ and knowing of a typical signal magnitude of 3 , the absolute measurement error $\sigma_{0}$ is about $1 \times 10^{-3}$. The worst case for the position resolution is at the 17-th measurement, where the orbit separation is the minimum value. The worst position resolution is

$$
\sigma_{x}=6 \mu m, \sigma_{y}=6 \mu m, \sigma_{u}=75 \mu m, \sigma_{v}=51 \mu m .
$$

\section{EFFECT OF PHASE DIFFERENCE ERROR}

The relative phase difference $\theta$ between the two beams is determined by the distance between the interaction point
Table 2: Diagonal elements of the covariant matrix

\begin{tabular}{r|rrrrrr}
$\mathrm{j}$ & $\mathrm{p}$ & $\mathrm{x}$ & $\mathrm{y}$ & $\mathrm{q}$ & $\mathrm{u}$ & $\mathrm{v}$ \\
\hline $\mathrm{l}$ & 2.5 & 13.6 & 9.8 & 7.9 & 2373.3 & 418.6 \\
2 & 4.2 & 19.9 & 16.0 & 11.8 & 4543.2 & 789.9 \\
3 & 2.2 & 13.1 & 9.6 & 7.0 & 2095.0 & 404.3 \\
4 & 1.5 & 11.7 & 6.7 & 4.9 & 1313.2 & 252.8 \\
5 & 2.2 & 13.1 & 9.6 & 7.0 & 2095.1 & 404.3 \\
6 & 3.1 & 13.5 & 8.0 & 10.0 & 2782.5 & 171.7 \\
7 & 6.1 & 15.1 & 10.5 & 17.5 & 5762.0 & 272.7 \\
8 & 2.5 & 13.0 & 8.1 & 8.2 & 2252.4 & 168.1 \\
9 & 1.5 & 12.1 & 6.1 & 5.4 & 1383.1 & 121.7 \\
10 & 2.5 & 13.0 & 8.1 & 8.2 & 2252.4 & 168.1 \\
11 & 1.5 & 13.6 & 10.5 & 4.9 & 1384.9 & 497.3 \\
12 & 2.3 & 18.7 & 20.4 & 7.4 & 2581.4 & 1162.5 \\
13 & 2.0 & 13.2 & 10.5 & 6.6 & 1875.4 & 524.6 \\
14 & 1.0 & 11.7 & 6.8 & 3.3 & 834.0 & 267.4 \\
15 & 1.1 & 13.0 & 9.9 & 3.7 & 983.3 & 447.3 \\
16 & 2.7 & 15.9 & 16.3 & 8.2 & 2904.2 & 1127.6 \\
17 & 3.9 & 33.6 & 39.7 & 11.3 & 5637.0 & 2555.6 \\
18 & 2.5 & 15.0 & 15.1 & 7.7 & 2672.5 & 1070.1 \\
19 & 1.8 & 11.7 & 8.5 & 5.7 & 1657.1 & 557.2 \\
20 & 2.5 & 15.0 & 15.1 & 7.7 & 2672.5 & 1070.1 \\
21 & 1.5 & 13.6 & 10.5 & 4.9 & 1385.0 & 497.3 \\
22 & 2.3 & 18.7 & 20.4 & 7.4 & 2581.2 & 1162.4 \\
23 & 1.1 & 13.0 & 9.9 & 3.7 & 983.3 & 447.3 \\
24 & 1.0 & 11.7 & 6.8 & 3.3 & 834.0 & 267.4 \\
25 & 2.0 & 13.2 & 10.5 & 6.6 & 1875.4 & 524.6 \\
\hline
\end{tabular}

and the BPM, and must be kept constant for stable collisions. Even though the BPM is installed at the designed position within a few $\mathrm{mm}$, the relative phase may differ from the design value by several degrees. Simulation studies show that a major effect of the phase error is movement of the position reading in the horizontal direction. Figure 2 shows the position reading of two rings when each beam stays at the design position and the phase difference is changed around the nominal phase of $\pi / 4$. The phase error effect is more harmful in HER than in LER. If the position displacement is required to be less than $0.5 \mathrm{~mm}$ the phase error must be kept less than 1 degree.

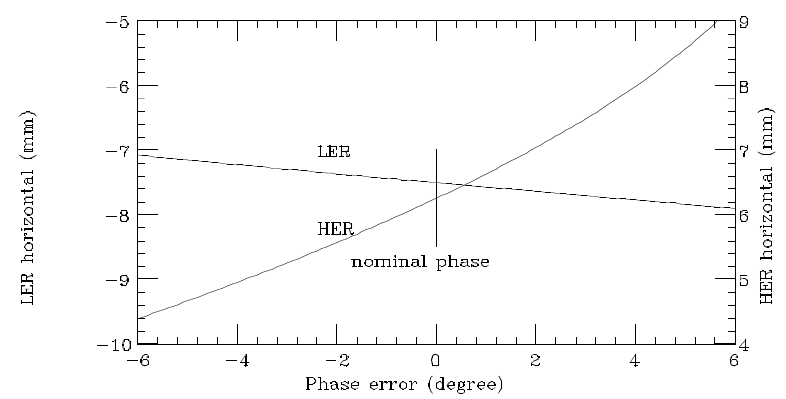

Figure 2: Position reading of two beams staying at the design orbit when the phase changes from the nominal value.

If the relative phase error exists, the position readings are also moved as the beam current changes. This fact helps finding the true phase difference. The position reading of HER is shown in Fig.3 for the case where the phase error is \pm 1 degree, each beam stays at the design orbit, and the electron charge of HER is increased from 0 to -1.6 while the positron charge of LER is fixed at 2.29, the nominal value. If the closed orbit of HER at the BPM is stable within $0.5 \mathrm{~mm}$ in real beam operations, we can find the 
true phase with a resolution of less than 0.5 degrees.

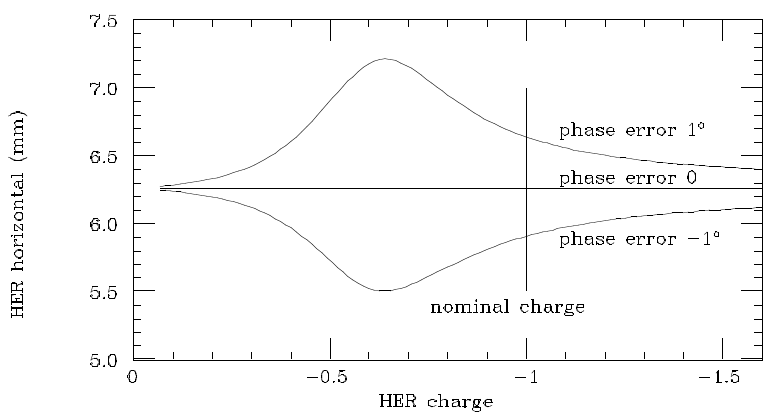

Figure 3: Position reading of HER when the phase error exists and the HER charge is changed.

The other effects of the phase error are deformation of the position sensitivity and the coupling, in which the position reading of one beam is moved when the other beam moves. These are analyzed by a simulation where one beam is fixed at the design orbit and the other beam is displaced from the design orbit within $2 \mathrm{~mm}$ in both the horizontal and vertical directions, and the phase error is $0.5 \mathrm{de}-$ grees. The two effects are shown in Fig. 4 when the HER orbit is fixed, and in Fig.5 for the other case. The grid indicates the ideal position reading including the displacement shown in Fig. 2 due to the phase error, and helps observe the deformation of the sensitivity. The crosses in the smaller box show the position reading of the fixed beam, and their distribution measures the coupling. The simulation shows that both effects are small, compared with the beam size at the BPM, particularly in the vertical direction. This fact favors the operation condition that the orbit stability is required in the vertical direction rather than in the horizontal direction.

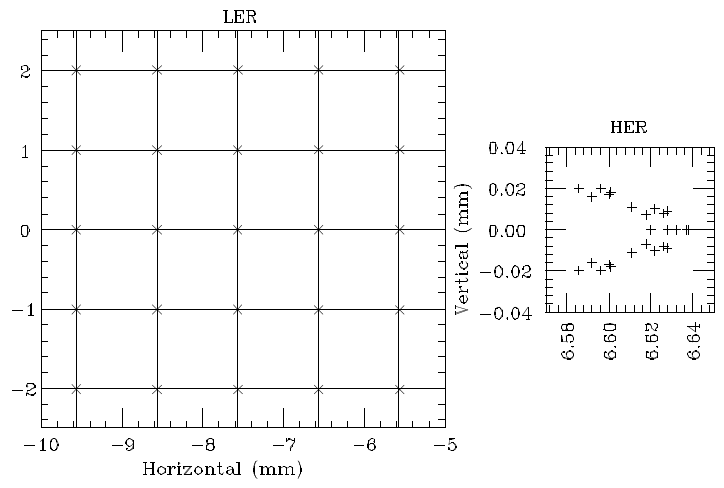

Figure 4: Position reading of two beams when the HER orbit is fixed and the other LER orbit is shifted.

\section{GAIN CALIBRATION}

So far the gains have been set unity in the paper. The gains of the real BPM are not equal to unity, and must be calibrated. Otherwise the monitor center would be shifted and
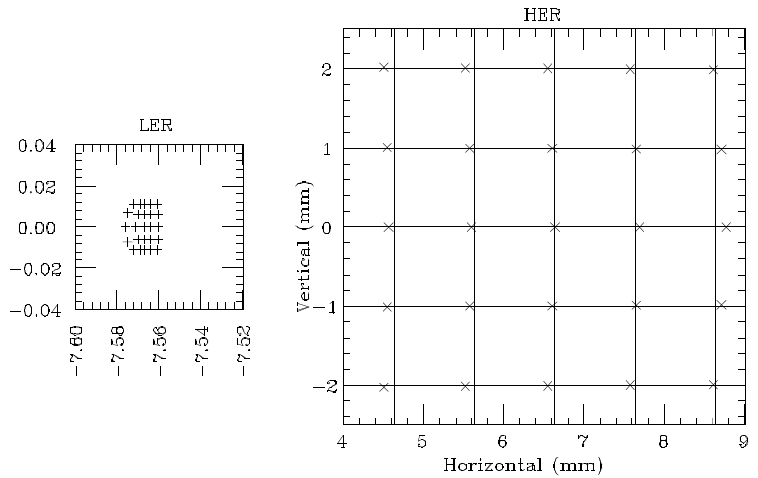

Figure 5: Position reading of two beams when the LER orbit is fixed and the other HER orbit is shifted.

the position sensitivity would be deformed. The gain calibration can be done with beams by another nonlinear fitting. The BPM has 8 electrodes and the number of unknown gains, normalized by the first electrode gain, is 7 . The gain calibration was studied under the condition that measurments are done 6 times with a single beam, 3 times for one beam and 3 times for the other. At each measurement the beam is on the design orbit, or displaced by $2 \mathrm{~mm}$ in the vertical direction. Beam measurement parameters and their covariant matrix elements together with elements for 7 relative gains are shown in Table 3. Assuming the relative detection error of $3 \times 10^{-4}$, we find that the gain error is less than $0.1 \%$, and the position resolution is less than $4 \mu \mathrm{m}$ in both directions.

Table 3: 6 beam positions and the giagonal elements of the covariant matrix for the gain fitting.

\begin{tabular}{r|rrrrrrr}
$\mathrm{j}$ & $\mathrm{p}$ & $\mathrm{x}$ & $\mathrm{y}$ & $\mathrm{C}(\mathrm{p})$ & $\mathrm{C}(\mathrm{x})$ & $\mathrm{C}(\mathrm{y})$ & \\
\hline 1 & 1.10 & -7.50 & -2.0 & 2.3 & 159 & 153 & \\
2 & 1.10 & -7.50 & 0 & 2.2 & 165 & 158 & \\
3 & 1.10 & -7.50 & 2.0 & 2.2 & 159 & 152 & \\
4 & 1.05 & 6.26 & -2.0 & 1.1 & 172 & 164 & \\
5 & 1.05 & 6.26 & 0 & 1.0 & 178 & 170 & \\
6 & 1.05 & 6.26 & 2.0 & 0.9 & 172 & 165 & \\
\hline \hline & $\mathrm{g} 2$ & $\mathrm{~g} 3$ & $\mathrm{~g} 4$ & $\mathrm{~g} 5$ & $\mathrm{~g} 6$ & $\mathrm{~g} 7$ & $\mathrm{~g} 8$ \\
$\mathrm{C}(\mathrm{g})$ & 0.9 & 1.8 & 3.7 & 4.7 & 3.1 & 2.4 & 1.1 \\
\hline
\end{tabular}

\section{REFERENCES}

[1] K. SATOH and M. TEJIMA, Proceedings of the 1995 Particle Accelerator Conference, Dallas, 2482.

[2] K. SATOH and M. TEJIMA, Proceedings of the 1997 Particle Accelerator Conference, Vancouver, 2087. 